\title{
Spin Transport in Poly-Acene Films and the Derivative Films by Using the Spin Pumping
}

Yuji Tanaka, Taisei Kono, Yoshio Teki, Eiji Shikoh

\begin{tabular}{|c|l|}
\hline \multicolumn{1}{|c|}{ Citation } & IEEE Transactions on Magnetics, 55(2); 1400304 \\
\hline \multicolumn{1}{|c|}{ Issue Date } & $2019-02$ \\
\hline Date of Publication & 2018-08-17 \\
\hline \multicolumn{1}{c|}{ Type } & Journal Article \\
\hline Textversion & author \\
\hline Rights & $\begin{array}{l}\text { C 2018 IEEE. Personal use of this material is permitted. Permission from } \\
\text { IEEE must be obtained for all other uses, in any current or future media, } \\
\text { including reprinting/republishing this material for advertising or promotional } \\
\text { purposes, creating new collective works, for resale or redistribution to servers } \\
\text { or lists, or reuse of any copyrighted component of this work in other works. } \\
\text { The article has been published in final form at } \\
\text { https://doi.org/10.1109/TMAG.2018.2856275 } \\
\text { https://ieeexplore.ieee.org/document/8439078 }\end{array}$ \\
\hline DOI & \begin{tabular}{l} 
10.1109/TMAG.2018.2856275 \\
\hline
\end{tabular} \\
\hline
\end{tabular}

\author{
Self-Archiving by Author(s) \\ Placed on: Osaka City University
}

TANAKA, Y., KONO, T., TEKI, Y., \& SHIKOH, E. (2018). Spin Transport in Poly-Acene Films and the Derivative Films by Using the Spin Pumping. IEEE Transactions on Magnetics. 55, 1400304. 


\title{
Spin transport in poly-acene films and the derivative films by using the spin-pumping
}

\author{
Yuji Tanaka ${ }^{1}$, Taisei Kono ${ }^{1}$, Yoshio Teki ${ }^{2}$, Eiji Shikoh $^{1}$ \\ ${ }^{1}$ Graduate School of Engineering, Osaka City University, 3-3-138 Sugimoto, Sumiyoshi-ku, Osaka 558-8585, Japan \\ ${ }^{2}$ Graduate School of Science, Osaka City University, 3-3-138 Sugimoto, Sumiyoshi-ku, Osaka 558-8585, Japan
}

\begin{abstract}
The spin transport in thermally-evaporated tetracene films and 6,13-bis(triisopropylsilylethynyl) pentacene (TIPS-pentacene) films by using the spin-pumping-induced spin injection method was challenged at room temperature. The spin transport in the TIPSpentacene films was demonstrated, while the spin transport in tetracene films was not succeeded. For tetracene films, reconstruction of the prepared films was occurred due to the low vapor pressure and it might be caused the deformation of ferromagnetic material as the spin injector.
\end{abstract}

Index Terms-Organic, spin-pumping, Spintronics, transport

\section{INTRODUCTION}

Qpin transport experiments of various organic molecular

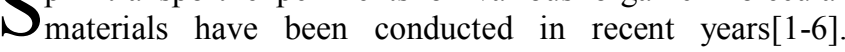

Previously, our group achieved spin transport in thermally evaporated pentacene films[1]. Carbon based organic semiconductors(OSs) such as pentacene and fullerene have small spin orbit interaction, so it is excellent as a transport material for pure spin current $[1,2,4]$. Therefore, we focused on tetracene and TIPS-pentacene which are similar properties to pentacene. Since the structures are similar, we investigate the transmission principle of pure spin current in OSs by comparing the transport properties and crystallinity of these. Both tetracene and TIPS-pentacene are p-type semiconductors and are known to be organic molecular materials showing high conductivity and high crystallinity $[7,8]$. The carrier mobility for the TIPSpentacene films is reported to be $6 \mathrm{~cm}^{2} \mathrm{~V}^{-1} \mathrm{~s}^{-1}$ due to the good crystallinity[7], and there is sufficient amount of active carriers to propagate the spin current. Although TIPS-pentacene films are often formed using a solution process[7-9], the spin coating method which is generally used is unsuitable for use in device fabrications. So in this study, all OSs are formed by vacuum thermal deposition. In addition, since pentacene, tetracene, and TIPS-pentacene all have photoconductivity such that electrical conductivity is increased by light irradiation with visible light[10-12], there is an advantage that development of a spinphotonic device is expected. In this study, we aim to achieve

Table. 1. Organic semiconductor and structure.

\begin{tabular}{|c|c|}
\hline Name & Molecular structure \\
\hline \multicolumn{2}{|l|}{ Pentacene } \\
\hline \multicolumn{2}{|l|}{ Tetracene } \\
\hline 6,13-bis-(triisopropylsilylethynyl)- & \\
\hline Pentacene(TIPS-pentacene) & \\
\hline
\end{tabular}

spin transport of TIPS-pentacene and tetracene films. We investigate the transport principle of the spin current in OSs from the characteristics of spin transport.

\section{EXPERIMENTAL METHOD}

Pure spin current is generated by the spin-pumping[13]. Spin transport in OSs by an electrical method is done[8], but there is a problem of conductance mismatch, which is related to the efficiency of spin injection, at the interface between ferromagnetic electrode and OS[14,15]. This problem is greatly suppressed by using the spin-pumping. Fig. 1 shows the sample structure in this study. $\mathrm{Ni}_{80} \mathrm{Fe}_{20}$ is used as a ferromagnetic metal (FM) which becomes a spin current injection source by ferromagnetic resonance(FMR)[16,17], and a pure spin current is transported in OSs and absorbed into Pd which is a nonmagnetic metal (NM). The inverse spin-Hall effect(ISHE) is generated by the spin orbit interaction of $\mathrm{Pd}$, the pure spin current is converted into a charge current, and it is detected as an electromotive force[18]. If we can prove that the electromotive force detected at Pd is due to the ISHE, it can be said that the spin transport in OSs have been achieved. When depositing NM / OSs / FM samples, the molecular structure may collapse in an electron beam evaporation method, so deposition of OSs is performed by resistance heating method[1]. All metals are deposited by an electron beam vapor deposition.

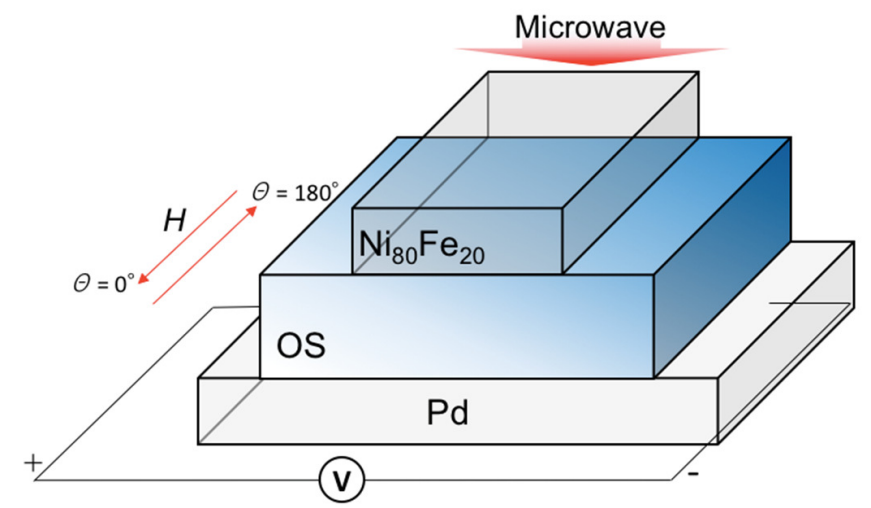

Fig. 1. Bird's-eye-view of the sample. $H$ is applied static magnetic field. 
All OSs were deposited at a rate of $1.0 \AA$ / s. Since the molecular structure may be destroyed due to the high energy of the metal when depositing the metal on the OS film, the substrate is cooled with cooling medium at $-2{ }^{\circ} \mathrm{C}$ and the vapor deposition is carried out at a low speed of $0.2 \AA / \mathrm{s}$. We used a microwave $\mathrm{TE}_{011}$-mode cavity in an electron spin resonance (ESR) system (JEOL, JES-TE300) to excite FMR in FM. The electromotive force is measured by using a nanovoltmeter (Keithley, 2182A), and the sample and the conductive wires are directly connected with a silver paste. In order to investigate the crystallinity of molecular material, the structure and surface analyses of the films were performed by an X-ray diffractometer (XRD) and an atomic force microscopy (AFM), respectively. All of the measurements were performed at room temperature.

\section{RESULT AND DISCUSSION}

FIG. 2(a) shows the FMR spectrum of a Pd / TIPS-pentacene / $\mathrm{Ni}_{80} \mathrm{Fe}_{20}$ sample at the thickness of TIPS-pentacene film $d=100$ $\mathrm{nm}$ at $\theta=0^{\circ}$. The FMR field $\left(H_{\mathrm{FMR}}\right)$ when FMR was excited was $1090 \mathrm{Oe}$ at the frequency of the microwave of $9.45 \mathrm{GHz}$ and the microwave power set to be $200 \mathrm{~mW}$. FIG. 2 (b) shows the electromotive force detected by Pd under the FMR. The circle is the measured value, and the curve is the fitting curve by the following equation[18-20]

$V(H)=V_{I S H E} \frac{\Gamma^{2}}{\left(H-H_{F M R}\right)^{2}+\Gamma^{2}}+V_{A s y m} \frac{-2 \Gamma\left(H-H_{F M R}\right)}{\left(H-H_{F M R}\right)^{2}+\Gamma^{2}}$,

where $\Gamma$ is the damping constant. The first and the second terms
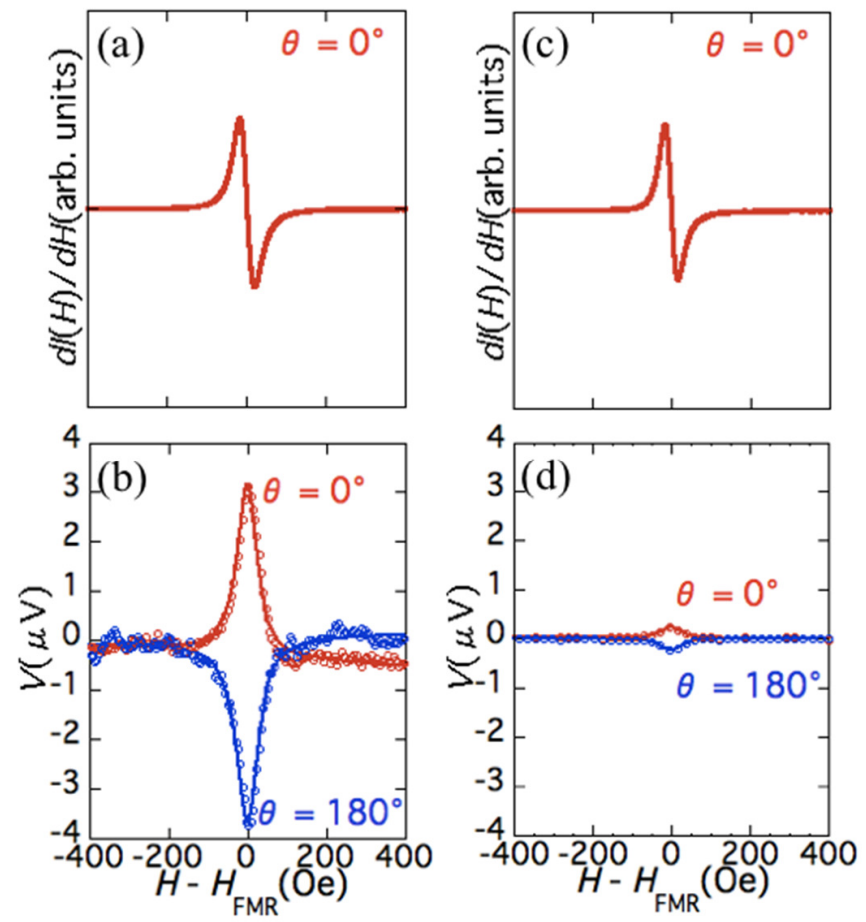

Fig. 2. (a) FMR spectrum of the sample using TIPS-pentacene. NM layer is Pd. Film thickness of TIPS-pentacene $d$ is $100 \mathrm{~nm}$. (b)Output voltage at $\operatorname{Pd}(\theta=0,180)$. (c) FMR spectrum of the sample with $\mathrm{Cu}$ and $d=100 \mathrm{~nm}$. (d) Output voltage at $\mathrm{Cu}(\theta=0,180)$ of the Eq. (1) represent the symmetric and the asymmetric components of the detected electromotive force to the $H_{\mathrm{FMR}}$, respectively. The asymmetric component is due to an effect such as anomalous Hall effect(AHE)[18-20]. The electromotive force was observed at the $H_{\mathrm{FMR}}$, and when the applied magnetic field was inverted, the output voltage was reversed. This is a characteristic of the electromotive force caused by the ISHE[18]. Figs. 2(c) and (d) are results of a control experiment in which $\mathrm{Pd}$ was changed to $\mathrm{Cu}$ which is known to have a small spin orbit interaction[21]. Other conditions are the same as in the case of Pd. Fig. 2 (d) shows that a small electromotive force is detected. This is understood to be the ISHE in the layer of copper oxide[22]. Therefore, it can be considered that the electromotive force detected by Pd as shown in Fig. 2(b) is due to the ISHE at Pd.

As another control experiment, the dependence of the electromotive force on the microwave power was investigated. Fig. 3 shows that the electromotive force depends on the microwave power. When the microwave power was varied from 0 to $200 \mathrm{~mW}$, the value of the electromotive force detected was proportional. This is also a significant result seen in the electromotive force caused by the spin-pumping and the ISHE[23]. From the above it can be said that spin transport in TIPS-pentacene film has been achieved. As discussed below, there is a possibility that the TIPS-pentacene film quality may be problematic.

Fig. 4 shows thickness dependence of $V_{\text {ISHE }}$ of TIPS-pentacene. The black line is curve fits under the assumption of exponential decay. The spin diffusion length is calculated from the relation of $V(d) \propto e^{-\frac{d}{\lambda_{s}}}[5]$, where $\lambda_{s}$ is the spin diffusion length of the thermally-evaporated TIPS-pentacene film. $\lambda_{s}$ was estimated to be $35 \mathrm{~nm} \pm 1 \mathrm{~nm}$. Compared with the spin diffusion length of pentacene, $42 \mathrm{~nm}[1], \lambda_{\mathrm{s}}$ is close value.

Fig. 5 shows the result of using tetracene as the second layer. The detected FMR intensity was weak and no clear
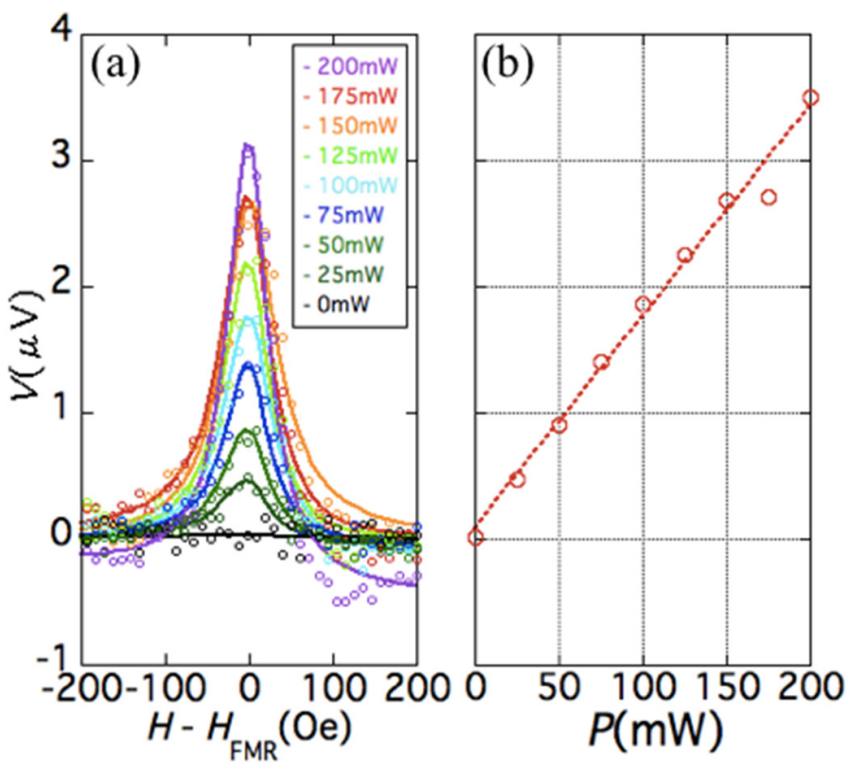

Fig. 3. (a)Microwave power dependence of electromotive force. Microwave power $(P)$ is changed $200 \mathrm{~mW}$ to $0 \sim 200 \mathrm{~mW}$. (b)Analysis results obtained with Eq. (1). $V$ correspond to the coefficients of the first term in Eq. (1). 


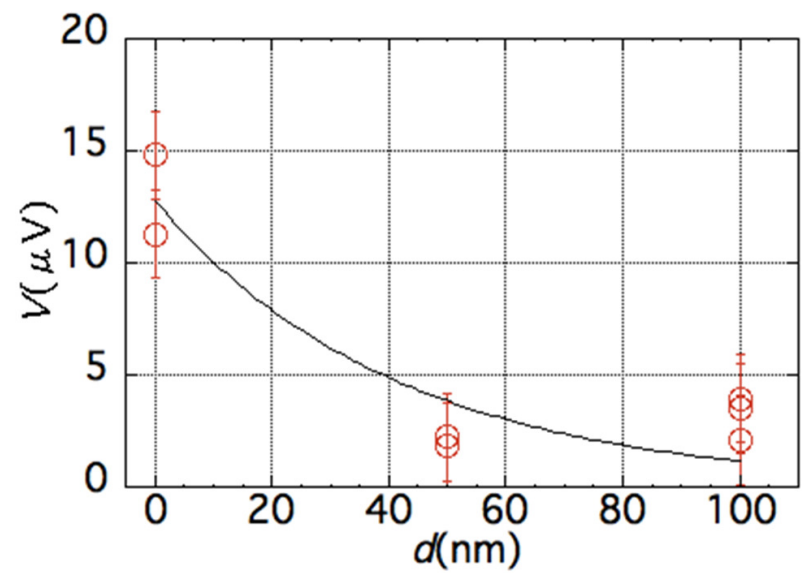

Fig. 4. Thickness dependence of $\mathrm{V}_{\text {ISHE }}$ of TIPS-pentacene $(d=0,50,100$ $\mathrm{nm})$. Open circles are the experimental data. The black line is curve fits under the assumption of exponential decay. Error bars are shown at each data point.

electromotive force was detected. It was considered that the $\mathrm{Ni}_{80} \mathrm{Fe}_{20}$ could not be a continuous film, and a sufficient amount of spin current was not generated.

In order to investigate the film quality problem, we investigated the crystallinity of OS films using XRD as shown in Fig. 6 with a previously obtained data for a pentacene film[1]. In respective samples, OSs were formed by vacuum thermal evaporation on a p-type Si substrate with an oxide film, and the wavelength of the $\mathrm{X}$ ray was $\mathrm{Cu}-\mathrm{K} \alpha$ radiation (wavelength $\lambda=0.154 \mathrm{~nm}$ ) was used in the XRD experiments. Pentacene and tetracene have diffraction peaks detected at respective certain angles[8, 24], whereas they are not detected with TIPS-pentacene. Therefore, the crystallinity of TIPS-pentacene films is not good. In the tetracene sample, a diffraction peak was detected, and consistent with the previous study[24]. But diffraction peak of tetracene film is smaller than that of pentacene. Therefore, it was concluded that the reason why the electromotive force was not detected is not the poor crystallinity of tetracene but the roughness of the interface. The surface roughness of a film for tetracene and for TIPS-pentacene are $70 \mathrm{~nm}$ and $13 \mathrm{~nm}$ by AFM measurements, where as the film thickness is $50 \mathrm{~nm}$ for both films. Compared to pentacene, it is known that the saturated vapor pressure of tetracene is higher than the vapor pressure for pentacene and weaker intermolecular interaction of tetracene, so that it can easily re-evaporate from the substrate in vacuum at room temperature[25]. Therefore, it is presumed that the surface of tetracene has become rough.

We thought that spin transport in tetracene film can be verified by reducing the film roughness. In addition, since the crystallinity may be related to the spin diffusion length, it can be said that the improvement of the crystallinity of TIPSpentacene is important in elucidating the mechanism of spin transport in OSs. In this study, measurement of electric conductivity of tetracene and TIPS pentacene is not carried out. Since further discussion is considered possible by comparing these values, measurements will be made in the future.

\section{SUMMARY}

In summary, spin transport in TIPS-pentacene films by thermal evaporation was achieved at room temperature. Compared to

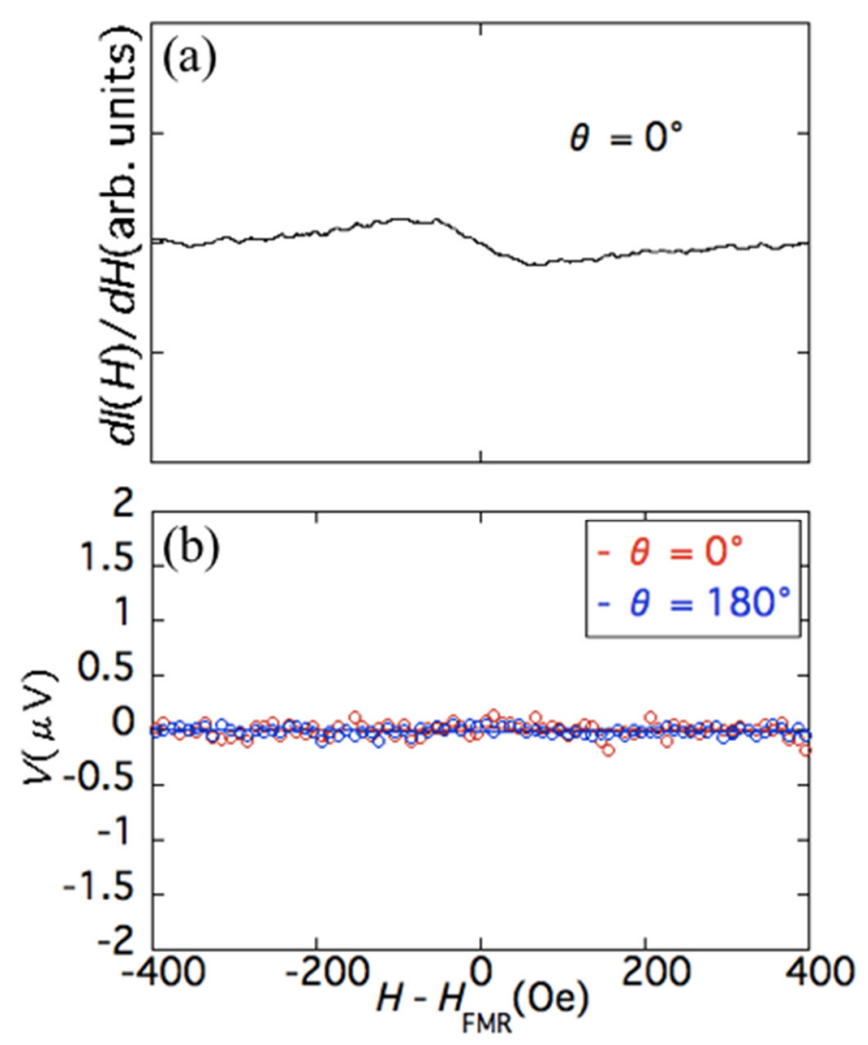

Fig. 5. (a) FMR spectrum of the sample using tetracene. Film thickness $d$ is $50 \mathrm{~nm}$. (b)Output voltage of sample with a Pd layer.

(a)

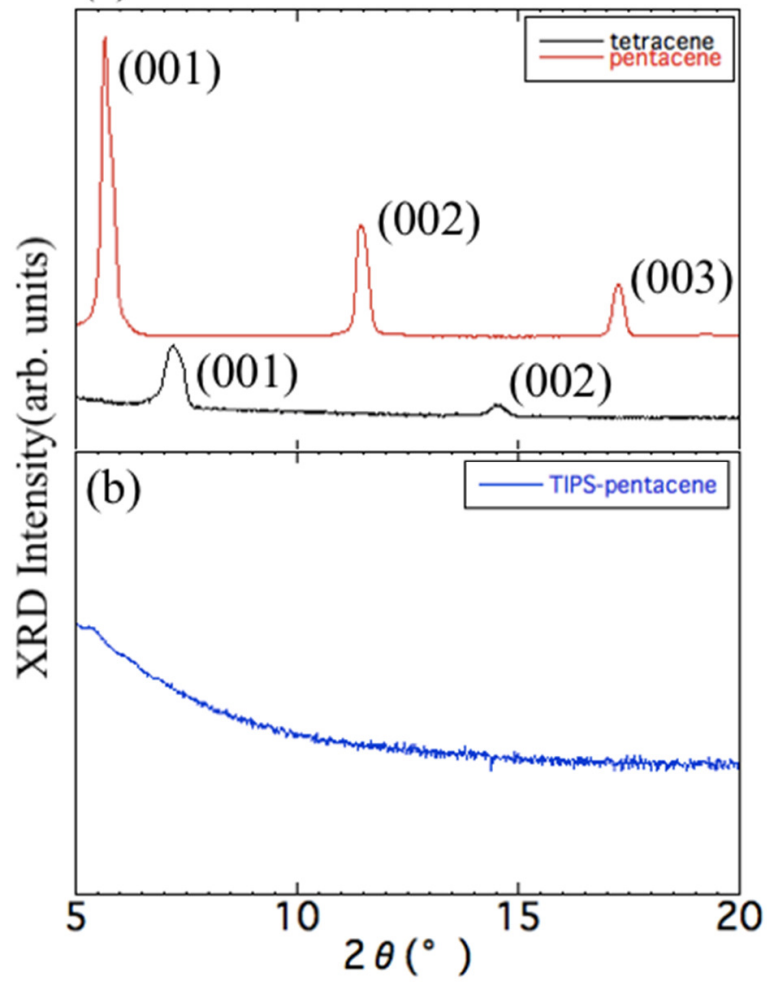

Fig. 6. XRD spectrum of OS films. All of film thickness of OS films are $100 \mathrm{~nm}$. (a)The XRD spectrum of pentacene and tetracene films. (b) XRD spectrum of a TIPS-pentacene films. 
the result of pentacle samples, there was a difference in electromotive force. Due to the roughness of the films, sufficient spin current was not generated for tetracene films, and no clear electromotive force was detected. $\lambda_{s}$ was estimated to be $35 \mathrm{~nm} \pm 1 \mathrm{~nm}$. As a result of examining the crystallinity of the OS, TIPS-pentacene are not uniform in crystalline state, and can be improved.

\section{ACKNOWLEDGMENTS}

This work was partly supported by JSPS Grants-in-Aid for Scientific Research(B) (16H04136) and by the Cooperative Research Program of "NJRC Mater. \& Dev.".

\section{REFERENCES}

[1] Y. Tani, Y. Teki, and E. Shikoh, "Spin-pump-induced spin transport in a thermally evaporated pentacene film.", Appl.Phys. Lett. 107, 242406(2015).

[2] M. Ohishi, M. Shiraishi, R. Nouchi, T. Nozaki, T. Shinjo, and Y. Suzuki, "Spin relaxation in graphene with self-assembled cobalt porphyrin molecules", Jpn. J. Appl. Phys., Part 2 46, L605(2007).

[3] J. B. S. Mendes, O. Alves Santos, J. P. Gomes, H. S. Assis, J. F. Felix, R. L. Rodriguez-Suarez, S. M. Rezende, and A. Azevedo, "Efficient spin transport through polyaniline", Phys. Rev. B 95, 014413(2017)

[4] HL. Liu, JY. Wang, M. Groesbeck, X. Pan, C. Zhang, ZV. Vardeny, "Studies of spin related processes in fullerene C-60 devices", J. Mater. Chem. $C, 614,3621-3627(2018)$.

[5] S. Watanabe, K. Ando, K. Kang, S. Mooser, Y. Vaynzof, H. Kurebatashi, E. Saitoh, and H. Sirringhaus, "Polaron spin current transport in organic semiconductors", Nat. Phys. 10, 308(2014).

[6] B. B. Chen, S. Wang, S. W. Jiang, Z. G. Yu, X. G. Wang, H. F. Ding, and $\mathrm{D}$. $\mathrm{Wu}$, "The role of heavy metal ions on spin transport in organic semiconductors", New. J. Phys. 17, 013004(2015).

[7] K. Wang, R. Chen, F. Zhuang, C. Chen, S. Su, Y. Xiang, "Study of triisopropylsilyl pentacene thin film and its interfacial properties for device applications", Thin. Solid. Films. 584, 359-362(2015).

[8] S. Mooser, J. F. K. Cooper, K.K. Banger, J. Wunderlich, and H. Sirringhaus, "Spin injection and transport in a solution-processed organic semiconductor at room temperature", Phys. Rev. B 85, 235202(2012).

[9] S. K. Park, T. N. Jackson, J. E. Anthony, and D. A. Mourey, "High mobility solution processed 6,13-bis(triisopropyl-silylethynyl) pentacene organic thin film transistors", Appl. Phys. Lett 91, 063514(2007).

[10] O. Ostroverkhova, D. G. Cooke, S. Shcherbyna, R. F. Egerton, F. A. Hegmann, R. R. Tykwinski, and J. E. Anthony, "Bandlike transport in pentacene and functionalized pentacene thin films revealed by subpicosecond transient photoconductivity measurements", Phys. Rev. B 71, 035204 (2005).

[11] José. C.S. Costa, Ricardo. J. S. Taveira, Carlos. F. R. A. C. Lima, Adélio Mendes, Luis. M. N. B. F. Santos, "Optical band gaps of organic semiconductor materials", Opt. Mater. 58, 51-60(2016).

[12] J. Day, S. Subramanian, J. E. Anthony, Z. Lu, R. J. Twieg, and O. Ostroverkhova, "Photoconductivity in organic thin films : From picoseconds to seconds after excitation", J. Appl. Phys 103, 123715(2008).

[13] S. Mizukami, Y. Ando, and T Miyazaki, "Effect of spin diffusion on Gilbert damping for a very thin permalloy layer in $\mathrm{Cu} / \mathrm{permalloy} / \mathrm{Cu} / \mathrm{Pt}$ films", Phys. Rev. B 66, 104413(2002).

[14] G. Schmidt, D. Ferrand, L.W. Molenkamp, A.T. Filip, and B.J. van Wees, "Fundamental obstacle for electrical spin injection from a ferromagnetic metal into a diffusive semiconductor", Phys. Rev. B 62, R4790 (2000).

[15] A. Fert and H. Jaffres, "Conditions for efficient spin injection from a ferromagnetic metal into a semiconductor", Phys. Rev. B 64, 184420(2001).

[16] Y. Tserkovnyak, A. Brataas, and G. E. W. Bauer, "Enhanced Gilbert Damping in Thin Ferromagnetic Films", Phys. Rev. Lett. 88, 117601(2002).

[17] K. Ando, S. Takahashi, J. Ieda, H. Kurebayashi, T. Trypiniotis, C. H. W. Barnes, S. Maekawa, and E. Saitoh, "Electrically tunable spin injector free from the impedance mismatch problem", Nat. Mater. 10, 655(2011).

[18] E. Saitoh, M. Ueda, H. Miyajima, and G. Tatara, "Conversion of spin current into charge current at room temperature Inverse spin-Hall effect", Appl. Phys. Lett. 88, 182509(2006).

[19] S. Dushenko, M. Koike, Y. Ando, T. Shinjo, M. Myronov, and M. Shiraishi, "Experimental Demonstration of Room-Temperature Spin Transport in n-Type Germanium Epilayers", Phys. Rev. Lett. 114, 196602(2015).

[20] K. Ando, and E. Saitoh, "Inverse spin-Hall effect in palladium at room temperature", J. Appl. Phys. 108, 113925(2010).
[21] H. L. Wang, C. H. Du, Y. Pu, R. Adur, P. C. Hammel, and F. Y. Yang, "Scaling of Spin Hall Angle in 3d, 4d, and $5 \mathrm{~d}$ Metals from $\mathrm{Y}_{3} \mathrm{Fe}_{5} \mathrm{O}_{12} /$ Metal Spin Pumping", Phys. Rev. Lett 112, 197201(2014).

[22] H. An, Y. Kageyama, Y. kanno, N. Enishi, and K. Ando, "Spin-torque generator engineered by natural oxidation of $\mathrm{Cu}$ ", Nat. Commun. 7, 13069(2016).

[23] Y. Kitamura, E. Shikoh, Y. Ando, T. Shinjo, M. Shiraishi, "Vertical spin transport in $\mathrm{Al}$ with $\mathrm{Pd} / \mathrm{Al} / \mathrm{Ni}_{80} \mathrm{Fe}_{20}$ trilayer films at room temperature by spin pumping", Sci. Rep. 3, 1739(2013)

[24] P. S. Abthagir, Y. Ha, E. You, S. Jeong, H. Seo, and J. Choi, "Studies of Tetracene- and Pentacene-Based Organic Thin-Film Transistors Fabricated by the Neutral Cluster Beam Deposition Method", J. Phys. Chem. B 109, 2391823924(2005).

[25] J. Shi, X. R. Qin, "Nucleation and growth of tetracene films on silicon oxide", Phys. Rev. B 78, 115412(2008) 